\title{
Long Noncoding RNA PVT1 Promotes Stemness and Temozolomide Resistance through miR-365/ELF4/SOX2 Axis in Glioma
}

\author{
Rui Gong, Zhi-Qiang Li, Kai Fu, Chao Ma, Wei Wang and Jin-Cao Chen* \\ Departments of Neurosurgery, Zhongnan Hospital of Wuhan University, Wuchang 430071, Hubei, P.R. China
}

\begin{abstract}
Long non-coding RNA (lncRNA) are a class of non-coding RNAs demonstrated to play pivotal roles in regulating tumor progression. Therefore, deciphering the regulatory role of lncRNA in the development of glioma may offer a promising therapeutic target for treatment of glioma. We performed RT-qPCR analysis on the expression of IncRNA plasmacytoma variant translocation 1 (PVT1) and miR-365 in glioma tissues and cell lines. Cell proliferation and viability was assessed with CCK8 assay. Cell migration was assessed by wound healing assay. Transwell assay was used to assess cell invasion capacity. Expression of CD133+ cells was detected by flow cytometry. Western blot assay was used to detection the expression of ELF4 and stemness-related protein SOX2, Oct4 and Nanog. Bioinformatics and dual-luciferase assay were used to predict and validate the interaction between PVT1 and miR-365. Elevated PVT1 expression was observed in glioma tissues and cells. Knockdown of PVT1 and overexpression of miR-365 inhibited proliferation, migration, invasion and promoted stemness and Temozolomide (TMZ) resistance of glioma cells. PVT1 regulated ELF4 expression by competitively binds to miR-365. PVT1 regulated the stemness and sensitivity of TMZ of glioma cells through miR-365/ELF4/ SOX2 axis. This study identified that PVT1 promoted glioma stemness through miR-365/ELF4/SOX2 axis.
\end{abstract}

Key words: IncRNA PVT1, miR-365, ELF4, SOX2, Glioma

\section{INTRODUCTION}

As one of the most common and aggressive malignant primary brain tumor, malignant glioma has invariably poor 5-y survival rates, due to invasive growth and high recurrence rate [1]. Therapeutic strategies for glioma are still limited because the molecular mechanisms underlying glioma formation still remain elusive [2]. Glioma displays strong infiltrating and aggressive properties [1]. The first therapeutic choice is surgery, followed by the treatment with the alkylating agent, Temozolomide (TMZ) [3] .

Cancer stem-like cells (CSCs) are functionally defined as a

Submitted November 3, 2020, Revised March 5, 2021,

Accepted March 7,2021

* To whom correspondence should be addressed.

TEL: 86-13971091714, FAX: 86-13971091714

e-mail: chenjincao7612@163.com subpopulation of cells within a tumor that can self-renew, have tumorigenic potential and can recapitulate the original tumor, which can enable them to persist, contributing to post-treatment recurrence [4]. The glioma malignancy is also increased by the presence of CSCs, which contributes to the aggressiveness, frequent relapse and higher resistance to chemotherapy and radiotherapy of glioma [5]. In glioma stem cells (GSCs), there is clonal heterogeneity at the genetic level with distinct tumorigenic potential, which is likely to influence disease progression and response to treatment [6]. In the past, several strategies have been pursued to target GSCs, such as induction of apoptosis, inhibition of self-renewal and chemoresistance-related pathways, or induction of their differentiation [7]. However, the regulatory mechanism of stemness and therapy resistance in GSCs remains largely unknown.

E74 Like ETS Transcription Factor 4 (ELF4) is a member of Ets family of proteins [8]. It has been proposed to function as a oncogene in some contexts, contributing to tumor formation [9]. It 
has also been reported that ELF4 is highly expressed in gliomas and contributes to glioma genesis by promoting stem cell traits, through direct activation of Sox 2 expression [10].

Long noncoding RNAs (lncRNAs) are a class of non-coding RNAs longer than 200 nucleotides without coding potential [11]. Accumulating evidences demonstrated that they play pivotal role in the regulation of a variety of cellular biological process such as cell proliferation, differentiation, metastasis and drug resistance [12-15]. Recent studies also have showed that the lncRNA plasmacytoma variant translocation 1 (PVT1) is an oncogene in a variety of cancers including glioma. For example, PVT1 knockdown inhibits glioma progression by regulating miR-424 expression [16]. PVT1 also promotes glioma cell proliferation and invasion by targeting miR-200a [17]. Besides, downregulation of PVT1 can attenuate paclitaxel resistance in glioma cells by regulating miR-365 [18]. However, the mechanism of how PVT1 regulates stemness and therapy resistance in glioma remains unclear.

Recently, competitive endogenous RNA (ceRNA) has been reported as a new underlying mechanism of lncRNA, which lncRNAs act as miRNA sponges to regulate the expression of target of those miRNAs [19]. MiRNAs are small RNAs with approximately 22 nucleotides in length and act as gene expression regulators involved in the regulation of sophisticated gene expression to coordinate a broad spectrum of biological processes including carcinogenesis [20]. miR-365 was reported to be related with the development of many types of cancers. It is related to the occurrence and development of non-small-cell lung cancer (NSCLC) by inhibiting TRIM25 expression [21]. miR-365-3p/EHF/keratin 16 axis regulates oral squamous cell carcinoma metastasis [22]. While miR-365 promotes lung carcinogenesis by downregulating the USP33/SLIT2/ROBO1 axis [23]. Recently, it also has been reported to inhibit proliferation, migration and invasion of glioma by regulating ELF4 [24]. However, less is known about the role of miR-365-mediated regulation of stemness and TMZ resistance in glioma as well as its underlying mechanism.

In this study, we investigated the role and mechanism of lncRNA PVT1 in stemness and TMZ resistance of glioma. We showed that lncRNA PVT1 was upregulated in glioma tissues and cells and promoted stemness and TMZ resistance of glioma through miR365/ELF4 axis. These findings provided a new insight of the regulatory role of lncRNA in the development of glioma and offered a promising therapeutic target for treatment of glioma.

\section{MATERIALS AND METHODS}

\section{Tissue samples}

Glioma tissue specimens $(\mathrm{n}=30)$ and normal brain tissue speci- mens ( $\mathrm{n}=28$ ) were provided by the Zhongnan Hospital of Wuhan University. All patients' tissue specimens were promptly frozen in liquid nitrogen and maintained at $-80^{\circ} \mathrm{C}$ for the isolation of RNA and proteins later. No patients had ever received any treatment from neurosurgery, chemotherapy, or radiotherapy. The present study has been reviewed and approved by the Ethics Committee of Zhongnan Hospital of Wuhan University (IRB No. 20190319), and adhered to the tenets of the Declaration of Helsinki. Before participating in this study, each patient provided written informed consent. The patient's detailed characteristics are supplemented in Table 1.

\section{Cell lines and cell culture}

Human astrocyte normal cells HEB and the human glioma cell lines T98G, A172, U87, U251 and U373 obtained from American Type Culture Collection (ATCC, USA). Cells were cultured at $37^{\circ} \mathrm{C}$ in the presence of $5 \% \mathrm{CO}_{2}$ in high-glucose Dulbeccos modified Eagle's medium (DMEM, Gibco, USA) containing 10\% fetal bovine serum (FBS, Gibco, USA), $100 \mathrm{U} / \mathrm{ml}$ penicillin (HyClone, USA), and $100 \mu \mathrm{g} / \mathrm{ml}$ streptomycin (HyClone, USA). In cytotoxicity test, the U251 and U87 cells were exposed to increasing concentrations $(10,25,50,100,200 \mu \mathrm{M})$ of TMZ (Sigma-Aldrich, USA) for $48 \mathrm{~h}$.

\section{RNA extraction and RT- $q P C R$}

RNA from tissues and cells was extracted by TRI-reagent (Sigma, USA) according to the manufacturer's protocol. Then the RNA was measured with a NanoDrop-2000 spectrophotometer (Thermo Fisher Scientific, Waltham, MA). First-strand cDNA was

Table 1. Correlation between PVT1 expression and clinical feature of gliomas patients

\begin{tabular}{|c|c|c|c|c|}
\hline \multirow{2}{*}{ Variable } & \multirow{2}{*}{$\mathbf{n}$} & \multicolumn{2}{|c|}{ PVT1 expression } & \multirow{2}{*}{$\begin{array}{c}\text { p-value } \\
\text { (t test) }\end{array}$} \\
\hline & & Low & High & \\
\hline Age (years) & & & & $\mathrm{p}>0.05$ \\
\hline$<50$ & 17 & 9 & 8 & \\
\hline$\geq 50$ & 13 & 6 & 7 & \\
\hline Gender & & & & $\mathrm{p}>0.05$ \\
\hline Male & 18 & 11 & 7 & \\
\hline Female & 12 & 5 & 7 & \\
\hline KPS & & & & $\mathrm{p}>0.05$ \\
\hline$<70$ & 20 & 11 & 9 & \\
\hline$\geq 70$ & 10 & 5 & 5 & \\
\hline Tumor size & & & & $\mathrm{p}<0.05$ \\
\hline$<3 \mathrm{~cm}$ & 17 & 6 & 11 & \\
\hline$\geq 3 \mathrm{~cm}$ & 13 & 3 & 10 & \\
\hline Tumor grade & & & & $\mathrm{p}<0.01$ \\
\hline $\mathrm{I} \sim \mathrm{II}$ & 13 & 3 & 10 & \\
\hline III IV & 17 & 3 & 14 & \\
\hline
\end{tabular}


Table 2. Oligonucleotide primer sets for RT-qPCR

\begin{tabular}{llc}
\hline Name & \multicolumn{1}{c}{ Sequence $\left.\mathbf{( 5}^{\prime} \rightarrow \mathbf{3}^{\prime}\right)$} & p-value $(\mathbf{t}$ test $)$ \\
\hline PVT1 F & AAAACGGCAGCAGGAAATGT & 20 \\
PVT1 R & GGAGTCATGGGTGTCAGACA & 20 \\
GAPDH-F & CCAGGTGGTCTCCTCTGA & 18 \\
GAPDH-R & GCTGTAGCCAAATCGTTGT & 19 \\
miR-365-F & CAGGGACTTTTGGGGGCA & 18 \\
miR-365-R & GTCGTATCCAGTGCAGGGTCCGAGGTATTCGCACTGGATACGACCACA & 51 \\
ELF4-F & AGCTGTCACTCTGCCCAACT & 20 \\
ELF4-R & GGGTCAGTGACAGGTGAGGT & 17 \\
U6 F & CTCGCTTCGGCAGCACA & 20 \\
\hline
\end{tabular}

then synthesized with PrimeScript RT Master Mix System (Takara, China) following the manufacturer's protocol. Real-time qPCR of the reverse transcription products was determined with SYBR Green qPCR assay (Takara, China), analyzed through the 7500 Real-time PCR System (ABI, USA) and normalized with GAPDH or U6, respectively. The relative expression levels of RNAs were calculated using the comparative $2^{-\Delta \Delta \mathrm{CT}}$ method. All experiments were performed at least three times. The primers used for RTqPCR are listed in Table 2.

\section{Cell transfection}

miR-365 mimics, miR-365 inhibitor and their respective negative control (NC) were synthesized and purchased from GenePharma (Genepharma, China). The sh-RNA specific for PVT1 and shRNA (negative control) were designed and synthesized by Ribobio (Ribobio, Guangzhou, China). U87 and U251 cells were transfected with sh-PVT1 (100 pmol), miR-365 mimics (50 nM) and miR-365 inhibitor (100 nM) using Lipofectamine 2000 reagent (Invitrogen) in accordance with the manufacturer's instructions. Briefly, the cells were seeded in 6-well plates, 24 h later the cells were transfected according to the manufacturer's protocol. The RNA extraction and western blot experiment were conducted at $48 \mathrm{~h}$ after transfection.

\section{Transwell invasion assays}

The invasive ability of cells was determined using 24-well transwell chambers coated with Matrigel (BD Biosciences, USA). In brief, the upper chambers were added with $200 \mu \mathrm{l}$ serum-free DMEM, while the DMEM in the lower chamber was added $10 \%$ FBS. $1 \times 10^{5}$ transfected cells in serum-free medium were seeded at in the top chamber and incubated at $37^{\circ} \mathrm{C}$ in a humidified incubator containing $5 \% \mathrm{CO}_{2} .48 \mathrm{~h}$ after incubation, the non-invaded cells on the upper surface of the membrane were removed with a cotton swab, Cells on the lower part of the membrane were fixed with 5\% glutaraldehyde in phosphate-buffered saline (PBS) for $15 \mathrm{~min}$, fixed with $70 \%$ ethanol for $30 \mathrm{~min}$ and stained with $0.1 \%$ crystal violet for $10 \mathrm{~min}$. The invasive cells were quantified by photographing 3 independent visual fields under the microscope (Olympus, Japan).

\section{Wound-healing assay}

Wound-healing assay was conducted as described before. Briefly, cells were grown to $80 \%$ confluence in a 6-well plate with DMEM supplemented with $10 \%$ FBS and were scratched with sterile $10 \mu \mathrm{l}$ pipette tip to create a wound. Then, cells were washed with PBS to remove floating cells and added to a medium without serum for $48 \mathrm{~h}$. Photos of the wound were captured at different time points $(0$ and $24 \mathrm{~h}$ ) under $\times 100$ magnitude microscope.

\section{Sorting of $\mathrm{CD}_{133^{+}}$cells by flow cytometry}

Sorting of CD $133^{+}$cells was carried out as described before [25]. Briefly, cells were washed three times with PBS, collected by centrifugation at $300 \times \mathrm{g}$ for $5 \mathrm{~min}$ at room temperature, and resuspended and dissociated into single cell suspension in the PreSort Buffer (BD FACS ${ }^{\mathrm{TM}}$, USA) by repeatedly pipetting and were then centrifuged at $300 \times \mathrm{g}$ for $5 \mathrm{~min}$ at room temperature. Then, cells were washed with $8 \mathrm{ml}$ PBS, blocked with $2 \mathrm{ml} \mathrm{FcR} \mathrm{Blocking}$ Reagent (Miltenyi Biotec GmbH, Germany) for $10 \mathrm{~min}$ at $4^{\circ} \mathrm{C}$ and PE-conjugated anti-CD133 antibodies (1:1,000; Miltenyi Biotec $\mathrm{GmbH}$, Germany) for $10 \mathrm{~min}$ at $4^{\circ} \mathrm{C}$ in the dark. The cells were washed once with $2 \mathrm{ml}$ PBS and then the proportion of CD133 cells were detected using the BD FACSCalibur ${ }^{\mathrm{TM}}$ analyzer (BD FACS $^{\mathrm{TM}}$, USA) with FlowJo 7.6 software.

\section{Western blot}

Cells were harvested and isolated with RIPA lysis buffer (ThermoFisher Scientific, USA) containing protease inhibitors (Roche, Switzerland). Then, equal amounts of protein samples were separated by $10 \%$ SDS-PAGE gel electrophoresis and then transfer to PVDF membranes, and incubated with primary antibodies: ELF4 (Abcam, USA, 1:1,000), SOX2 (Abcam, USA, 1:1,000), Oct4 (Abcam, USA, 1:1,000), Nanog (Abcam, USA, 1:1,000) and GAPDH 
(Abcam, USA, 1:3,000). The membrane was then washed with TBST containing $0.1 \%$ Tween 20 for three times. Moreover, the membrane was incubated with HRP-labeled goat anti-mouse/rabbit $\operatorname{IgG}(1: 5,000, \mathrm{ZDGB}-$ bio, China) for $2 \mathrm{~h}$ at room temperature. Signals were visualized with SuperPico chemiluminescent substrate (Pierce, USA) and film exposure.

\section{Cell viability assay}

Cell viability was measured with a Cell Counting Kit 8 (CCK8) Kit (Dojindo, Japan). Briefly, $2 \times 10^{3} /$ well of cells were seeded into 96-well plates at $48 \mathrm{~h}$ after transfection. Then, $10 \mu \mathrm{l}$ of CCK8 solution was added into the culture medium, and the cells were incubated for $1 \mathrm{~h}$ at $37^{\circ} \mathrm{C}$. The OD value was read at $490 \mathrm{~nm}$ with a Microplate reader (BioRad, USA). The data are presented as means \pm SD of multiple experiments.

\section{Plasmid construction and luciferase activity assay}

The predicted miR-365 binding site on PVT1 and the 3' UTR of ELF4 as well as the mutated target region were amplified and cloned into the pmirGLO plasmids (Promega, USA). Then, 800 $\mathrm{ng} /$ well of luciferase reporter plasmids and $50 \mathrm{pmol} /$ well miR365 mimics were co-transfected into HEK-293T cells with Lipofectamine 2000 reagent (Invitrogen). $48 \mathrm{~h}$ after transfection, Dual-
Luciferase Reporter Assay System (Promega, USA) was used to examine the relative luciferase activity.

\section{Statistical analysis}

All experiments in this study were repeated three times. All results of the experiment were statistically analyzed using Graphpad Prism 5 (Graphpad, USA). The results were presented as mean \pm Standard Deviation (SD). Statistical significance was analyzed using Student's t-test (two groups) or one-way ANOVA test and Pearson's correlation coefficient. Survival analysis was carried out with the log-rank test and Kaplan-Meier method. $\mathrm{p}<0.05$ was considered statistically significant.

\section{RESULTS}

\section{PVT1 was upregulated and miR-365 was down-regulated in glioma tissues}

First, we investigate the expression of lncRNA PVT1 and miR365 in 30 glioma tissue samples and 28 non-tumor tissues by RT-qPCR. As shown in Fig. 1A, lncRNA PVT1 was significantly upregulated, while, miR-365 was downregulated in glioma tissue samples compared with normal tissues $(\mathrm{p}<0.01$, Student's $\mathrm{t}$ test, Fig. 1B). In addition, Pearson correlation coefficients clearly
A

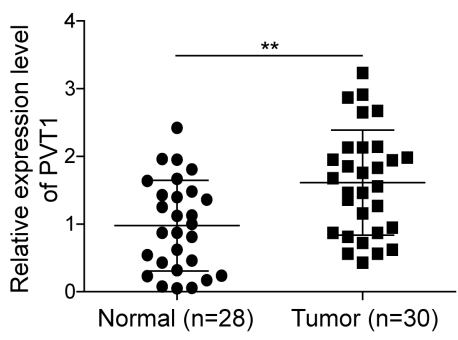

D

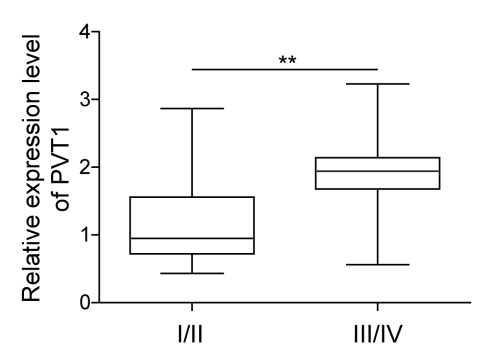

B

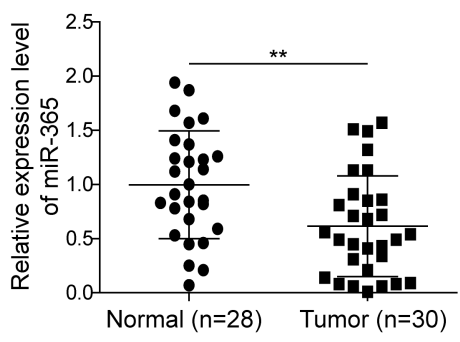

E

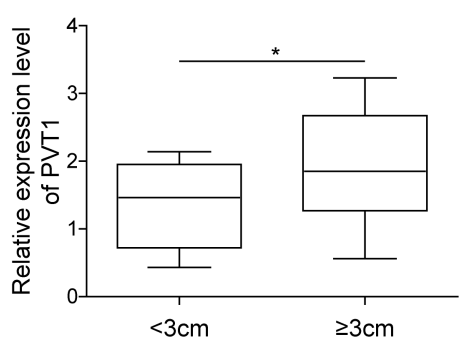

C

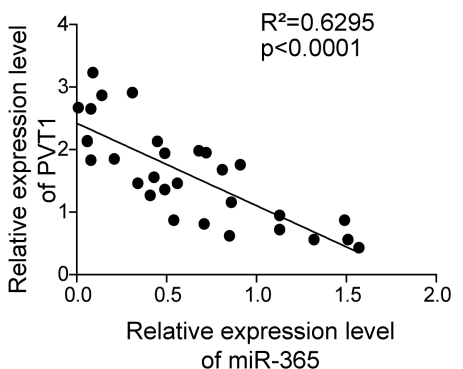

F

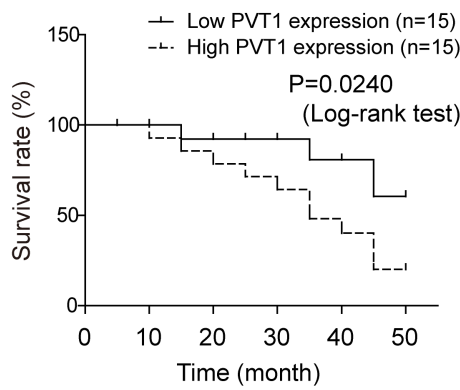

Fig. 1. PVT1 was upregulated and miR-365 was downregulated in glioma tissues. (A) The levels of lncRNA PVT1, assessed by RT-qPCR, were higher in glioma tissue samples comparatively to normal brain tissue specimens. GAPDH was used for normalization. (B) The expression of miR-365 detected by RT-qPCR, was down-regulated in glioma tissue samples compared with normal tissues. U6 was used for normalization. (C) Pearson's correlation test was used to verify correlations between PVT1 and miR-365, and analysis of the results revealed that expression of PVT1 was negtively correlated with miR365 in glioma tissue samples. (D) PVT1 expression, assessed by RT-qPCR, was positively correlated with the WHO grade and tumor size. (F) KaplanMeier survival curves revealed that high expression of PVT1 was associated with poor survival in glioma patients. Data are presented as Mean \pm SD of three independent experiments. ${ }^{*} \mathrm{p}<0.05,{ }^{* *} \mathrm{p}<0.01$. 
showed that the expression of PVT1 was negatively correlated with the expression of miR-365 ( $\mathrm{R}^{2}=0.6295, \mathrm{p}<0.001$, Fig. 1C). We also analyze the correlation between PVT1 expression and two clinicpathological parameters (WHO grade and tumor size). PVT1 was significantly associated with WHO grade (I II vs. III IV; $<<0.01$, Student's t-test, Fig. 1D) and tumor size ( $<3 \mathrm{~cm}$ vs. $\geq 3 \mathrm{~cm}$; $p<0.05$, Student's t-test, Fig. 1E) and not ralated with the patients age and gender ( $p>0.05$, Student's t-test,Table 1). Moreover, patients with high PVT1 expression levels exhibited markedly worse overall survival prognoses ( $\mathrm{p}<0.05, \log$-rank test, Fig. 1F).

\section{LncRNA PVT1 knockdown inhibited the stemness and TMZ resistance of glioma cells}

To explore the regulatory role of PVT1 on glioma cells, we then investigated the expression of PVT1 in glioma cell lines T98G, A172, U87, U251 and U373 as well as the human normal glial cell line HEB by RT-qPCR. As shown in Fig. 2A, PVT1 was significantly upregulated in glioma cells compared with HEB cells $(\mathrm{p}<0.05$, one-way ANOVA test), and the results of which showed that U87 and U251 cells showed the highest expression of PVT1 compare to other glioma cell lines. Then, U87 and U251 cells were transfected with PVT1 shRNA, as shown in Fig. 2B, PVT1 was successfully down-regulated in U87 and U251 cells transfected with sh-PVT1 compared with sh-NC ( $<<0.05$, Student's t-test). Cell proliferation, migration and invasion were inhibited in U87 and U251 cells transfected with sh-PVT1 compared with sh-NC $(\mathrm{p}<0.01$, Student's t-test, Fig. $2 \mathrm{C} \sim \mathrm{E})$. Moreover, the ratio of positive $\mathrm{CD} 133^{+}$cells was decreased after PVT1 knockdown (Fig. 2F). In addition, the expression of ELF4 and stemness factors (SOX2, Oct4, Nanog) of U87 and U251 cells were all decreased after PVT1 knockdown (Fig. 2G). Besides, effect of PVT1 knockdown on the sensitivity of U87 and U251 cells to TMZ was also evaluated, and sh-PVT1 could effectively promote the sensitivity of U87 and U251 cells to TMZ compared with sh-NC ( $\mathrm{p}<0.05$, Student's t-test, Fig. $2 \mathrm{H})$.

\section{miR-365 overexpression inhibited the stemness and TMZ resistance of glioma cells}

As the expression of miR-365 was proved to be negatively correlated with the expression of PVT1 in glioma tissues, we wonder whether it also has the opposite effect on the stemness of glioma cells. As shown in Fig. 3A, miR-365 was significantly upregulated in U87 and U251 cells transfected with miR-365 mimics compared with mimics- $\mathrm{NC}(\mathrm{p}<0.01$, Student's t-test). MiR-365 overexpression resulted in inhibition of cell proliferation, migration and invasion (Fig. 3B D, Student's t-test). Moreover, miR-365 overexpression also inhibited the ratio of $\mathrm{CD} 133^{+} \mathrm{U} 87$ and $\mathrm{U} 251$ cells
(Fig. 3E). In addition, the expression of ELF4 and stemness factors (SOX2, Oct4, Nanog) in U87 and U251 cells were all decreased after transfection with miR-365 mimics compared with mimics-NC $(\mathrm{p}<0.01$, one-way ANOVA test, Fig. 3F). Besides, effect of miR-365 overexpression on the sensitivity of U87 and U251 cells to TMZ was also evaluated, and miR-365 mimics could effectively promote the sensitivity of U87 and U251 cells to TMZ compared with sh$\mathrm{NC}(\mathrm{p}<0.01$, Student's t-test, Fig. 3G).

\section{PVT1 competitively binds miR-365 to regulate ELF4 expression}

To explore the mechanism by which PVT1 regulates stemness of glioma cells, we investigated the interaction among PVT1, miR365 and ELF4. First, the expression of miR-365 and ELF4 were detected in U87 and U251 cells after transfected with sh-PVT1 or sh-NC. MiR-365 was up-regulated ( $\mathrm{p}<0.01$, Student's t-test, Fig. 4A) while ELF4 was down-regulated after transfected with shPVT1 compare to sh- $\mathrm{NC}(\mathrm{p}<0.05$, Student's t-test, Fig. 4B). We also analyzied the correlation between the expression of PVT1 and ELF4 of glioma tissues. as shown in Fig. 4C, PVT1 and ELF4 was positively correlated in glioma tissue samples $\left(R^{2}=0.6659\right.$, $\mathrm{p}<0.001$ ), indicating ELF4 could be the downstream of PVT1. To further verify ELF4 was regulated by PVT1, ELF4 expression was detected in U87 cells after PVT1 knockdown by western blot, which showed that expression of ELF4 in U87 cells of sh-PVT1 group was significantly down-regulated as compared with that of the NC group ( $\mathrm{p}<0.01$, Student's t-test, Fig. 4D). Furthermore, to confirm PVT1 regulated ELF4 expression by competitively sponging the miR-365, the expression of PVT1 and ELF4 was detected in U87 cells after transfected with miR-365 mimics or inhibitor. As were shown in Fig. 4E, F, both PVT1 and ELF4 were down-regulated by miR- 365 overexpression and up-regulated by miR-365 inhibition. In addition, we predicted the potential miR365 binding region on PVT1 by bioinformatics (Fig.4G). Then, dual-luciferase reporters containing wild-type PVT1 (PVT1-wt) and mutant PVT1 (PVT1-mut) gene were constructed and their luciferase activity was detected by the dual-luciferase reporter assay. We demonstrated that miR-365 mimics significantly suppressed the luciferase activity of the luciferase reporter containing PVT1-wt in HEK-293T cells compared with mimics NC ( $p<0.05$, Student's t-test), and no effect observed on the mutated form (Fig. $4 G$ ), which indicated that PVT1 directly interacted with miR-365. Similarly, miR-365 mimics significantly suppressed the luciferase activity of the luciferase reporter containing ELF4 3'UTR-wt in HEK-293T cells compared with mimics NC ( $\mathrm{p}<0.01$, Student's $\mathrm{t}$ test), and no effect observed on the mutated form (Fig. 4H). Taken together, these results indicated that LncRNA PVT1 was a direct 

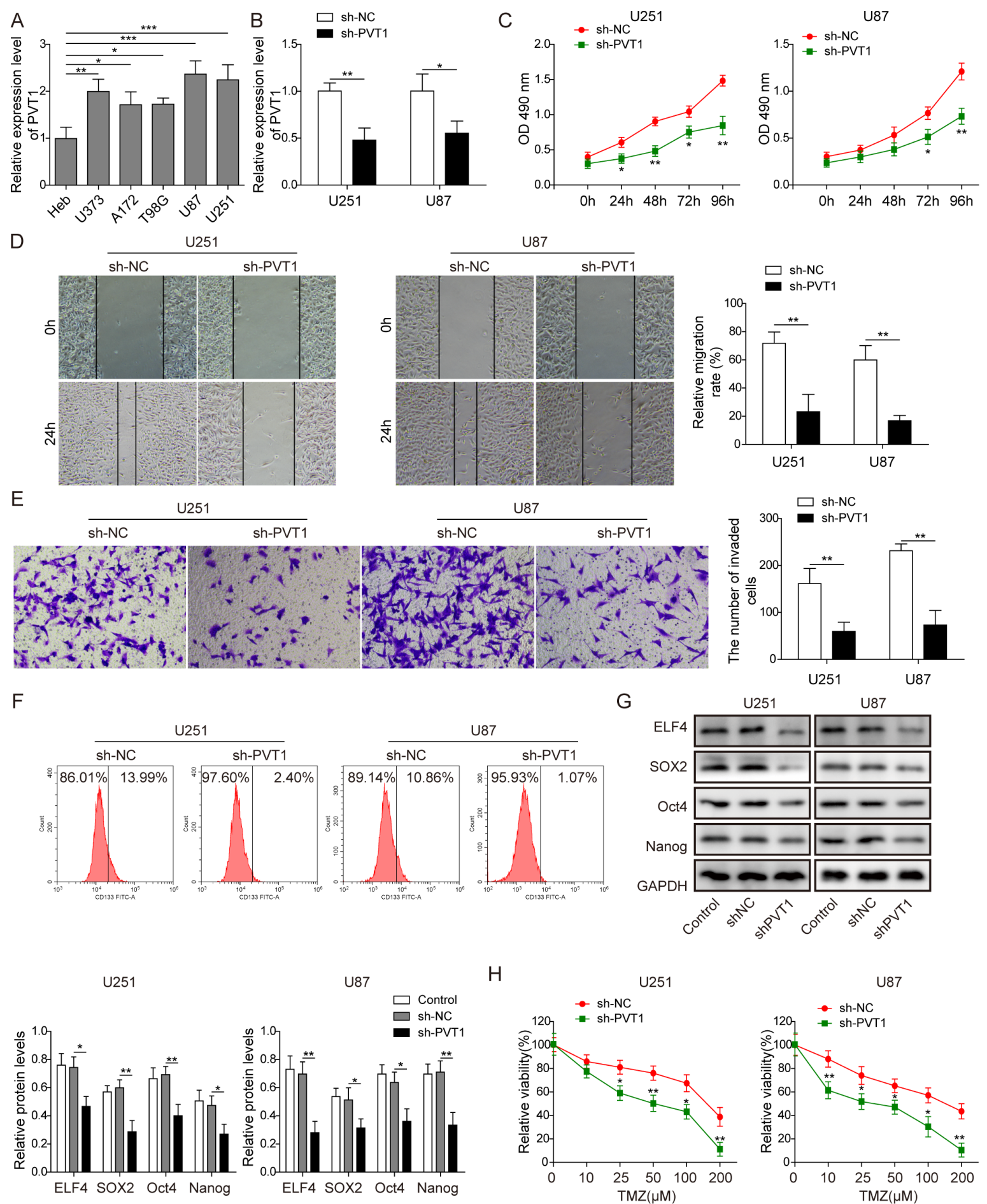

$\mathrm{H}$
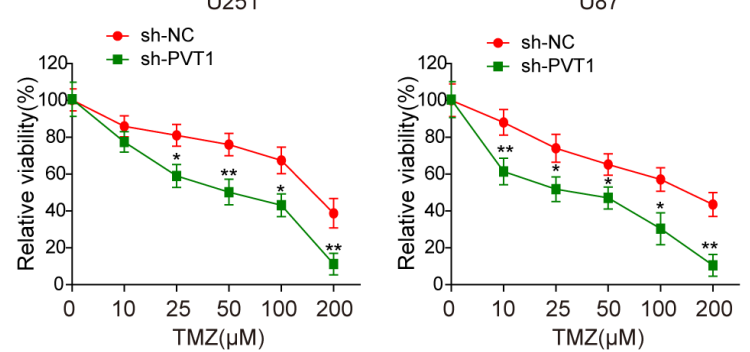

Fig. 2. PVT1 knockdown inhibited the stemness and TMZ resistance of glioma cells. (A) The expression level of PVT1 determined by RT-qPCR, was significantly up-regulated in glioma cells T98G, A172, U87, U251 and U373 compared with that in human astrocyte normal cells HEB. GAPDH was used for normalization. (B) The expression level of PVT1 in U87 and U251 cells after transfected with PTV1 shRNA and NC shRNA were detected by RT-qPCR. GAPDH was used for normalization. The results revealed that PVT1-shRNA expression vector was successfully constructed. (C) Cell proliferation was assessed by CCK8 and the results of CCK8 revealed that PVT1 knockdown inhibited glioma cell proliferation. (D) PVT1 knockdown inhibited glioma cell migration, as indicated by wound healing assay. (E) PVT1 knockdown inhibited glioma cell invasion, as indicated by transwell assay. (F) The rate of $\mathrm{CD}_{133^{+}} \mathrm{U} 87$ and U251 cells was suppressed by PVT1 knockdown, as indicated by flow cytometry assay. (G) The protein level of ELF4 and stemness factors (SOX2, Oct4, Nanog) in glioma cells were decreased after PVT1 knockdown, as indicated by western blot. GAPDH was used for normalization. (H) PVT1 knockdown inhibited resistance of U87 and U251 cells to TMZ, as detected by CCK8 assay. Data are presented as Mean \pm SD of three independent experiments. ${ }^{*} \mathrm{p}<0.05,{ }^{* *} \mathrm{p}<0.01,{ }^{* * *} \mathrm{p}<0.001$. 

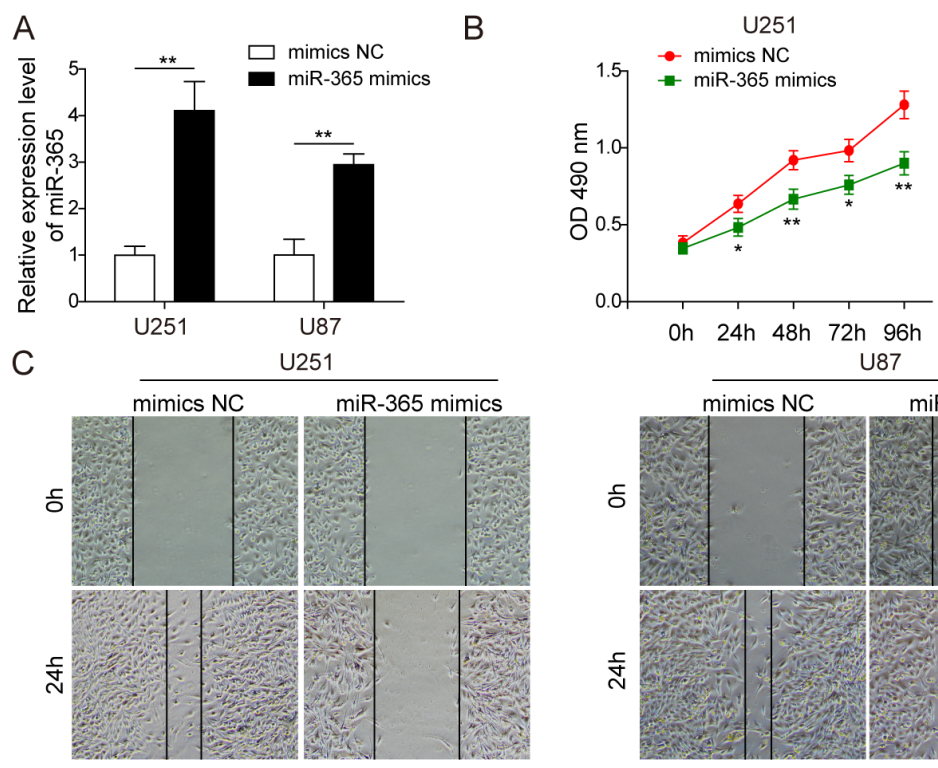

Oh $24 \mathrm{~h} 48 \mathrm{~h} 72 \mathrm{~h} \quad 96 \mathrm{~h}$

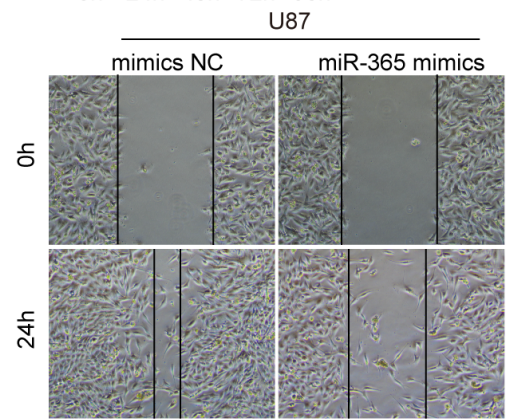

D

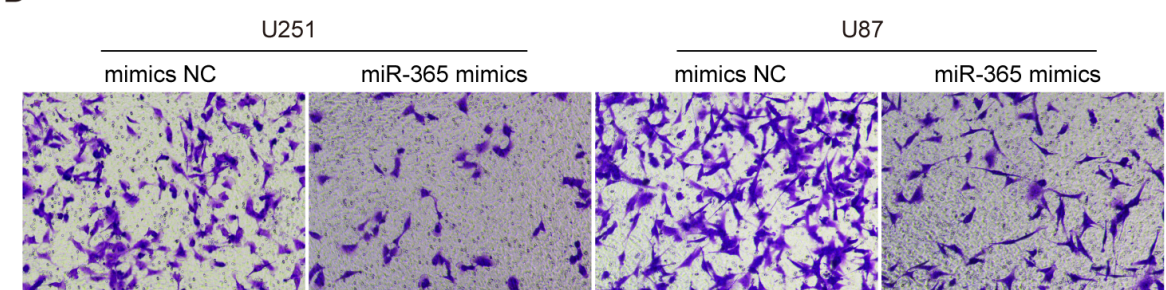

F

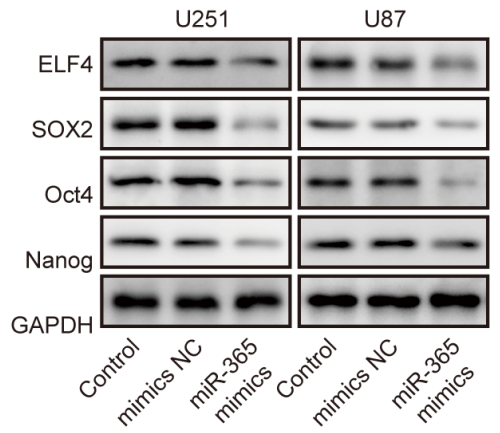

G
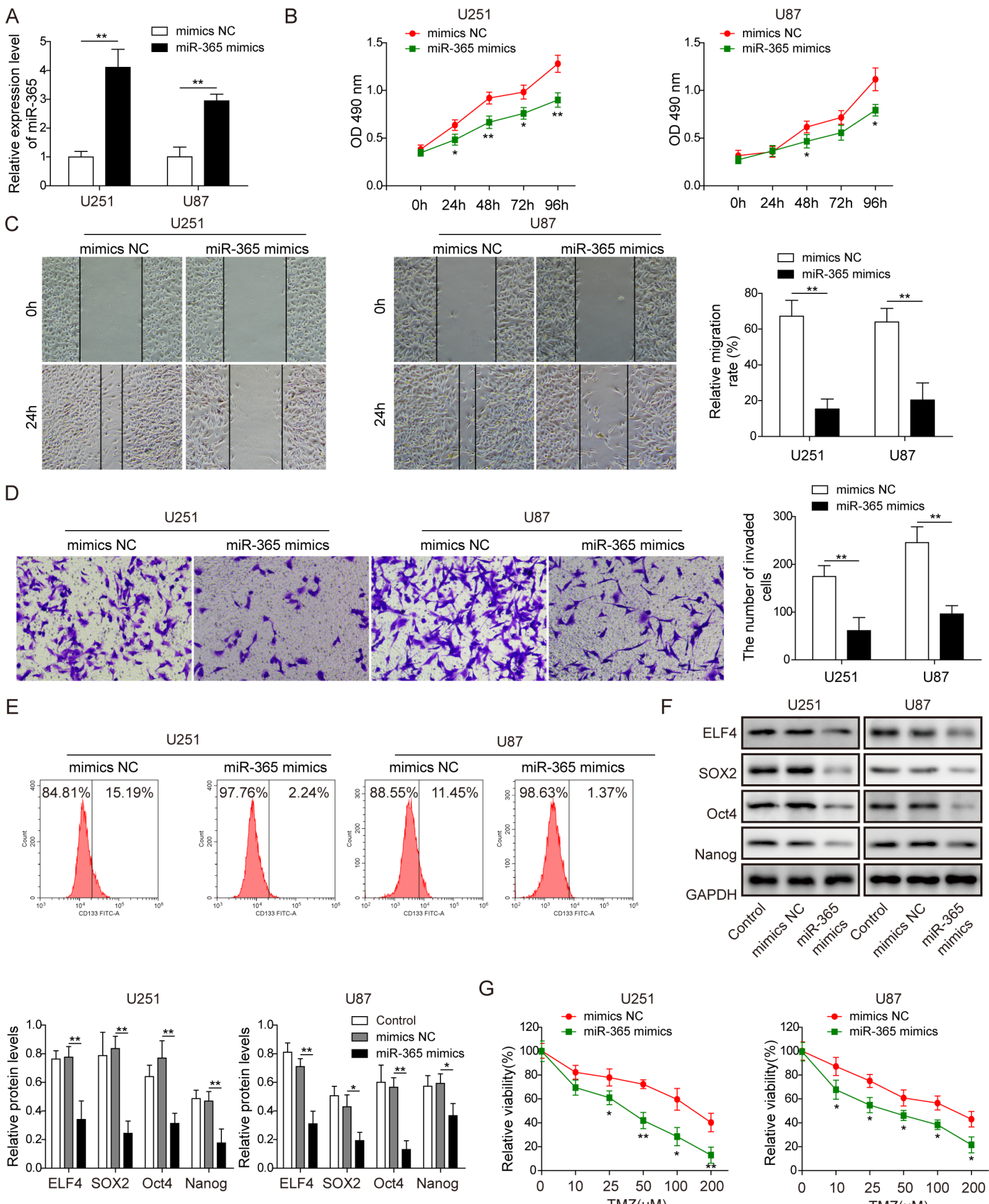

E
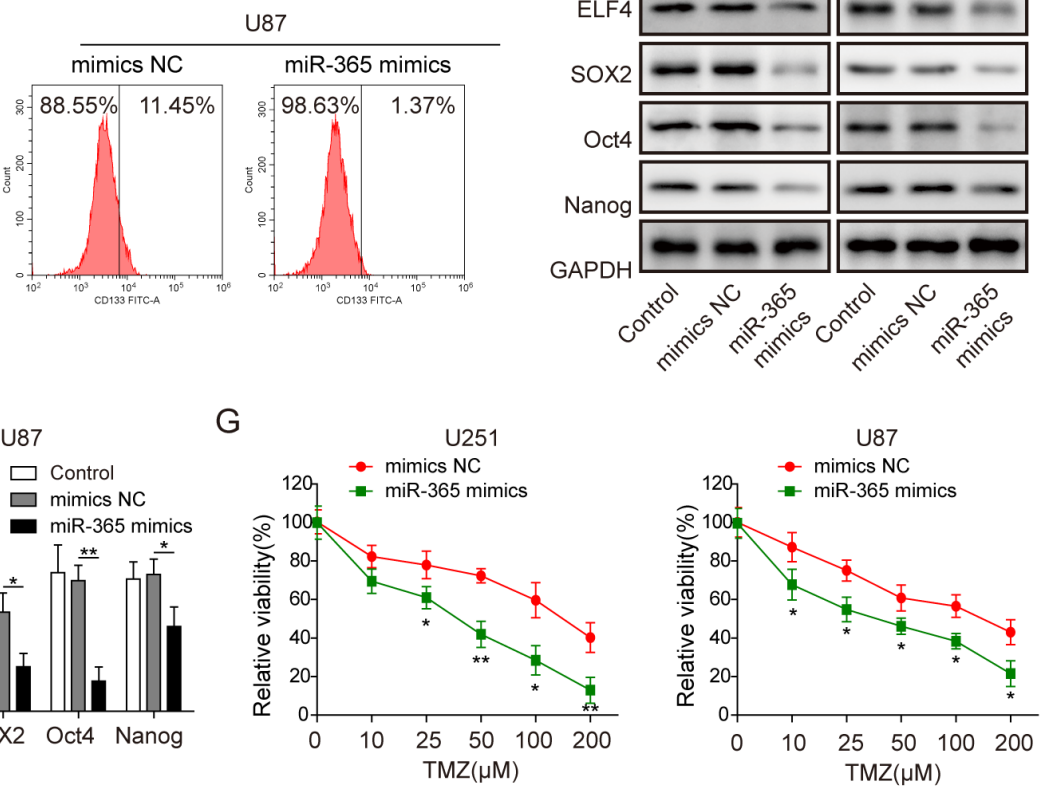

Fig. 3. miR-365 overexpression inhibited the stemness and TMZ resistance of glioma cells. (A) The expression level of miR-365 was successfully upregulated in U87 and U251 cells after transfected with miR-365 mimics, as detected by RT-qPCR. (B) miR-365 overexpression inhibited glioma cell proliferation, as indicated by CCK8. (C) MiR-365 overexpression inhibited glioma cell migration indicated by wound healing assay. (D) miR-365 overexpression inhibited glioma cell invasion, as shown by transwell assay. (E) The rate of CD133 ${ }^{+}$U87 and U251 cells was suppressed after miR-365 overexpression, as demonstrated by flow cytometry assay. (F) The protein level of ELF4 and stemness factors (SOX2, Oct4, Nanog) were inhibited by miR-365 overexpression in glioma cells, as indicated by western blot. GAPDH was used for normalization. (G) Overexpression of miR-365 reduced glioma cell viability and sensitized cells to TMZ, as detected by CCK8 assay. Data are presented as Mean \pm SD of three independent experiments. ${ }^{*} \mathrm{p}<0.05$, ${ }^{* *} \mathrm{p}<0.01$, ${ }^{* * *} \mathrm{p}<0.001$. 
A

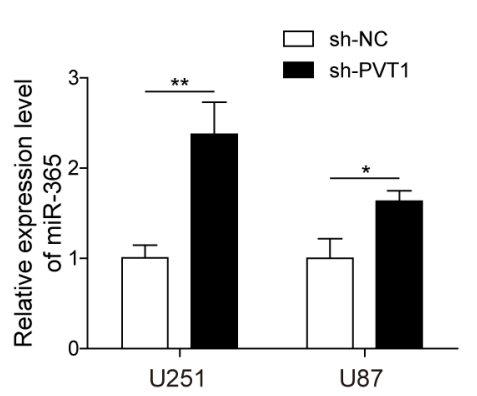

D
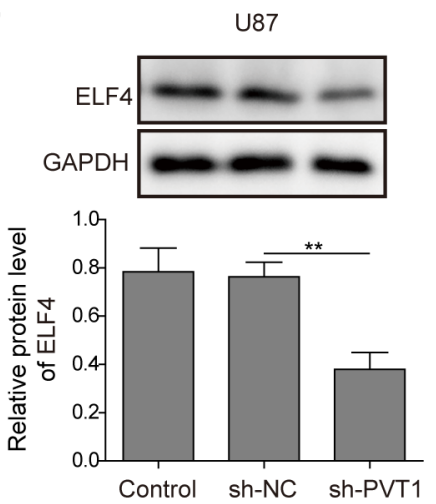

G
$B$

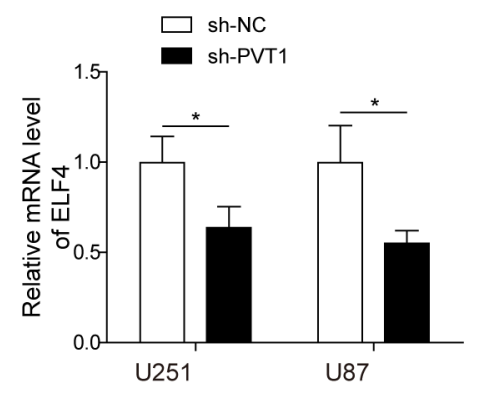

$E$

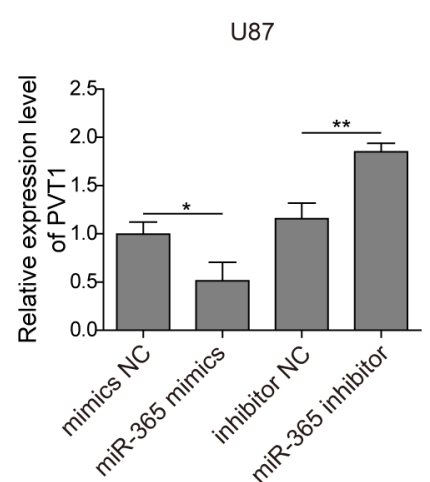

C

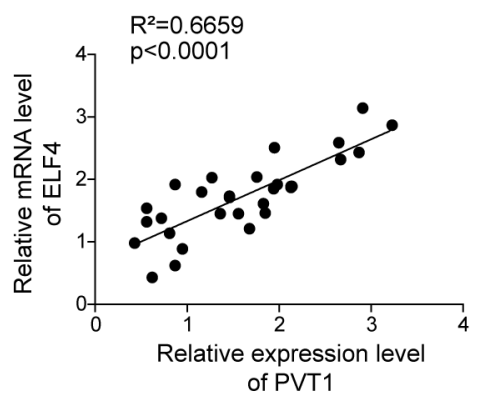

$\mathrm{F}$

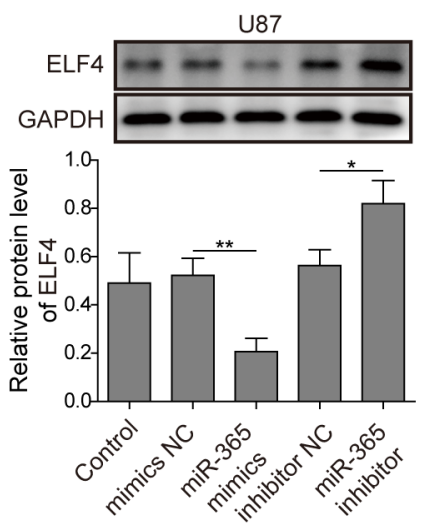

$\begin{array}{rr}\text { PVT1-WT } & \text { 5'...GGCUGAGUGACCUUGGGCAUU...3' } \\ \text { Hsa-miR-365 } & \text { 3' UAUUCCUAAAAAUCCCCGUAAU } 5 \\ \text { PVT1-MUT } & 5^{\prime} \text {...GGCUGAGUGACCUUGCCGUAA... }\end{array}$

$\mathrm{H}$

\author{
ELF4 3'UTR-WT $\quad 5^{\prime}$ '...GUGUUUCCAGCAGUUGGCAUUAA....' \\ Hsa-miR-365 UAUUCCUAAAAAUCCCCGUAAU 5 \\ ELF4 3'UTR-MUT 5'...GUGUUUCCAGCAGUUGGTGTUAA...3'
}
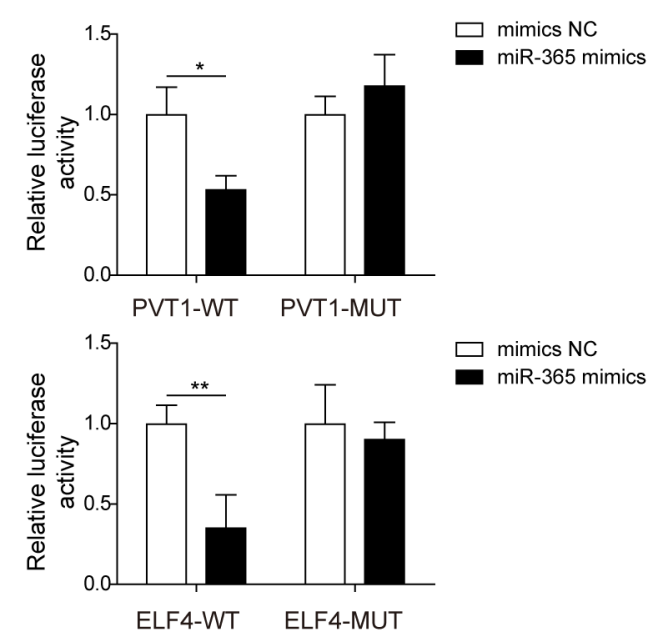

Fig. 4. PVT1 competitively binds miR-365 to regulate ELF4 expression. (A) Elevated miR-365 expression observed when PVT1 was knockdown in glioma cells U87 and U251, as detected by RT-qPCR. (B) ELF4 mRNA expression was downregulated when PVT1 was knockdown in glioma cells U87 and U251, as shown by RT-qPCR. (C) lncRNA PVT1 and ELF4 expression were positively correlated in glioma tissue samples, as analysed by Pearson's correlation test. (D) Protein level of ELF4 was downregulated when PVT1 was knockdown in glioma cells U87 and U251, as shown by western blot. GAPDH was used for normalization. (E) PVT1 expression was downregulated in U87 cells after transfected with miR-365 mimics and upregulated after transfected with miR-365 inhibitor, as shown by RT-qPCR. (F) mRNA and protein level ELF4 expression were down-regulated in U87 cells after transfected with miR-365 mimics and and upregulated after transfected with miR-365 inhibitor, as demonstrated by western blot. GAPDH was used for normalization. (G) Bioinformatics analysis predicted that PVT1 specifically targeted miR-365, which was validated by dual-luciferase reporter assay. (H) ELF4 was a target for miR-365, as verified by bioinformatics analysis and dual-luciferase reporter assay. Data are presented as Mean \pm SD of three independent experiments. ${ }^{*} \mathrm{p}<0.05,{ }^{* *} \mathrm{p}<0.01$.

target of miR-365 and positively modulated ELF4 expression by competitively binding to miR-365 in glioma cells.

\section{PVT1 regulated the stemness and TMZ resistance of glioma} cells through modulation of miR-365/ELF4/SOX2 axis

Next, we test whether PVT1 regulates stemness and TMZ resistance of glioma cells through miR-365/ELF4/SOX2 axis. As shown 
A

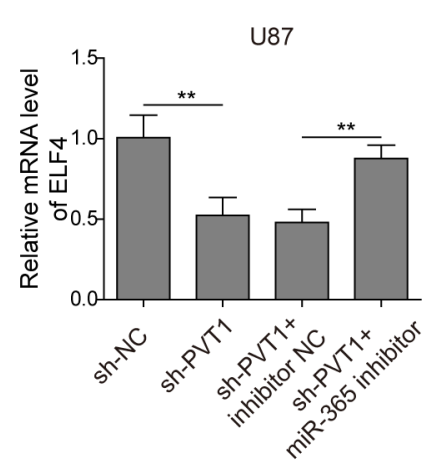

C

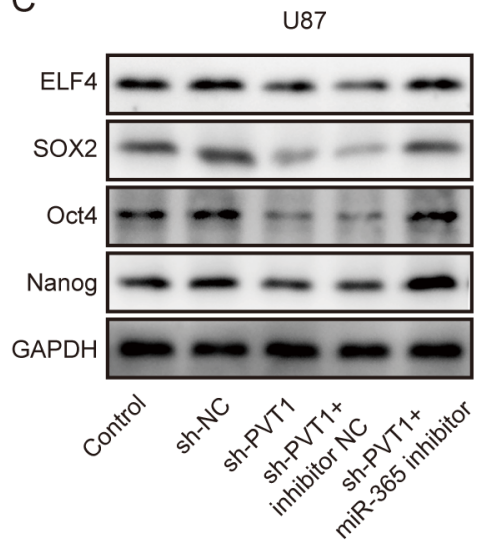

B

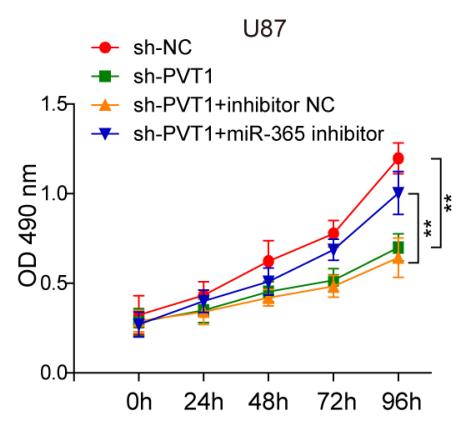

D

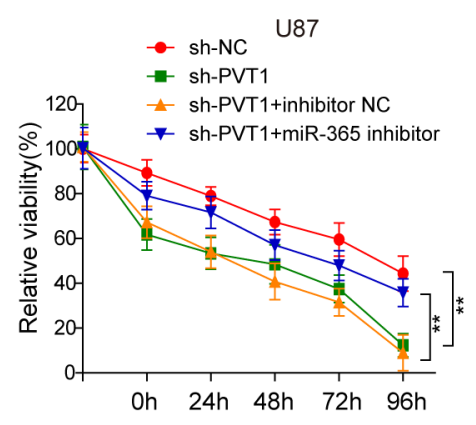

Fig. 5. PVT1 regulated the stemness and TMZ resistance of glioma cells through miR-365/ELF4/SOX2 pathway. (A) The expression level of ELF4 was downregulated by PVT1 knockdown, which was rescued by miR-365 inhibitor, as detected by RT-qPCR. (B) Cell proliferation was inhibited by PVT1 knockdown, which was rescued by miR-365 inhibitor, as indicated by CCK8. (C) The protein level of ELF4 and stemness factors (SOX2, Oct4, Nanog) were inhibited by PVT1 knockdown, which was rescued by miR-365 inhibitor in glioma cells, as shown by western blot. GAPDH was used for normalization. (D) PVT1 knockdown reduced glioma cell viability and sensitized cells to TMZ, which was rescued by miR-365 inhibitor, as measured by CCK8 assay. Data are presented as Mean $\pm \mathrm{SD}$ of three independent experiments. ${ }^{*} \mathrm{p}<0.05,{ }^{* *} \mathrm{p}<0.01$.

in Fig. 5A, ELF4 was down-regulated by PVT1 knockdown, while, miR-365 inhibitor rescued this effect compared with that of inhibitor-NC ( $p<0.01$, one-way ANOVA test). At the same time, PVT1 knockdown inhibited the proliferation of U87 cells, which could be significantly rescued by miR-365 inhibitor compared with inhibitor-NC (Fig. 5B). The expression of ELF4 and stemness factors SOX2, Oct4 and Nanog were also downregulated in the sh-PVT1 group compared with the sh-NC group ( $\mathrm{p}<0.01$, one-way ANOVA test). While, the effect of sh-PVT1 on the expression of ELF4 and stemness factors SOX2, Oct4 and Nanog was rescued by miR-365 inhibition (Fig. 5C). In addition, the elevated sensitivity of U87 to TMZ by PVT1 knockdown was also compensated by miR-365 inhibition (Fig. 5D). Taken together, these results indicated that PVT1 could regulate the stemness and TMZ resistance of glioma cells through miR-365/ELF4/SOX2 axis.

\section{DISCUSSION}

As the most common and aggressive primary central nervous system (CNS) tumors, gliomas exhibit poor prognosis due to tumor infiltration, treatment resistance and tumor recurrence [26]. GSCs, a small subpopulation of self-renewing and tumorigenic cancer stem cells, have been implicated in the unresponsiveness of glioma tumors to therapy and are most crucial for driving invasive tumor growth and relapse [6]. Thus, understanding the underlying molecular mechanisms of glioma stemness will help in the design of more effective therapies against glioma and preventing tumor recurrence. Besides, lncRNAs are emerging as important regulators of various biological processes. However, the specific functions of IncRNAs in GSCs are just beginning to be characterized, which needs further elucidation [27]. In this study, we demonstrated that lncRNA PVT1 was upregulated in glioma tissues and cells and promoted stemness and TMZ resistance of glioma.

Increasing evidence demonstrates that IncRNA plays regulatory 
role on the stemness of glioma cells. Studies have shown that lncRNA UCA1 is necessary for stemness via acting as a ceRNA for Slug in glioma cells [28]. While, increased level of H19 promotes invasion, angiogenesis, and stemness of glioma cells [29]. In the present study, we found that lncRNA PVT1 was upregulated in glioma tissues and cells, indicating that PVT1 may be involved in the tumorigenesis or progression of glioma. Additionally, we found that PVT1 knockdown inhibited the stemness of glioma cells and enhanced the sensitivity of glioma cells to TMZ. These results implied that PVT1 could help in the design of more effective therapies against glioma. In consistent with our results, many lncRNAs have been reported to be associated with development of TMZ resistance. LncRNA TP73-AS1 can promote temozolomide resistance in glioma cancer stem cells [30]. LncRNA AC003092.1 was also reported to promote TMZ chemosensitivity through miR195/TFPI-2 signaling modulation in glioma [31]. In this study, we found that PVT1 regulated the stemness and TMZ resistance of glioma cells through modulation of miR-365/ELF4/SOX2 axis. In addition, targeted nanocomplex carrying siRNA against MALAT1 was reported to sensitize glioma to temozolomide, which indicated combining standard TMZ treatment with lncRNA-targeting therapies by nanocomplex could enhance the very poor prognosis for glioma patients recently [15]. Thus, whether systemic delivery of PVT1 shRNA via a nanocomplex that targets brain tumors may enhance the therapeutic efficacy of TMZ should also be explored in the future.

Many studies have shown that lncRNA can function as ceRNA to sponge miRNAs thus to regulate the expression of their target genes $[32,33]$. In this study, bioinformatics and luciferase reporter assays were carried out and verified the interaction of lncRNA PVT1 and ELF4 with miR-365. Our data showed that miR-365 was significantly upregulated when PVT1 was knockdown, while, ELF4 expression was significantly downregulated when PVT1 was knockdown. As ELF4 was previously reported to be the target of miR-365 [34]. Therefore, we demonstrated that PVT1 promoted stemness and TMZ resistance and functions as ceRNA to regulate ELF4 expression by sponging miR-365 in glioma cells. Consistent with the previous report that PVT1 could suppress cell proliferation and invasion of colorectal cancer via sponging miR-214-3p [35]. PVT1 can promote tumor progression by regulating the miR143/HK2 axis in gallbladder cancer [36].

Moreover, luciferase reporter assays demonstrated that lncRNA PVT1 directly interacted with miR-365 and we also proved that miR-365 inhibited the stemness and sensitivity to TMZ of glioma cells. As no previous studies have reported that miR-365 could regulate drug sensitivity of glioma cells, this is the first study that uncover a regulatory role of miR-365 in the sensitivity of glioma cells to TMZ. Moreover, the expression of miR-365 was negatively correlated with the expression of ELF4 and direct binding of miR-365 to ELF4 was proved by dual-luciferase reporter assays. Thus, we concluded that lncRNA PVT1 positively regulates posttranscriptional expression of ELF4 through sponging miR-365.

Here, we also further implicated that PVT1 regulated the stemness of glioma cells though ELF4/SOX2 signaling pathway. SOX2, HMG-box transcription factor, plays an important role in maintaining stem cell self-renewal within glioma [37]. It also acted as an oncogene in gliomas [38] and was identified as a direct target of ELF4 in mouse primary brain cells and human glioma cell lines [10]. In our study, we validated that ELF4/SOX2 axis is downstream pathway of miR-365. Our data suggested that miR-365/ ELF4/SOX2 pathway may be responsible for the ability of PVT1 to modulate stem-like characteristics of glioma cells.

In conclusion, we showed that lncRNA PVT1 could enhance the stemness and TMZ resistance in glioma cells by serving as a ceRNA to regulate ELF4 expression by sponging miR-365 in glioma. This interaction would be considered a potential target for the diagnosis of glioma and outcome assays of TMZ-based therapy, thus, further elucidating the function of PVT1 is of much significance to explain the mechanism of which TMZ resistance and even can overcome the resistance in glioma. Besides, these findings may also provide a better understanding of the biological effects of IncRNA on stemness in glioma cells. Thus, understanding the underlying molecular mechanisms of glioma stemness will help in the design of more effective therapies against glioma and preventing tumor recurrence.

\section{ACKNOWLEDGEMENTS}

We would like to give our sincere gratitude to the reviewers for their constructive comments. This work was supported by the $\mathrm{Nu}$ trition group of Zhongnan Hospital of Wuhan University, China.

\section{CONFLICT OF INTEREST}

The authors declare that there is no conflict of interest.

\section{REFERENCES}

1. Davis ME (2016) Glioblastoma: overview of disease and treatment. Clin J Oncol Nurs 20(5 Suppl):S2-S8.

2. Cheng Z, Li Z, Ma K, Li X, Tian N, Duan J, Xiao X, Wang Y (2017) Long non-coding RNA XIST promotes glioma tumorigenicity and angiogenesis by acting as a molecular sponge of miR-429. J Cancer 8:4106-4116. 
3. Daniel P, Sabri S, Chaddad A, Meehan B, Jean-Claude B, Rak J, Abdulkarim BS (2019) Temozolomide induced hypermutation in glioma: evolutionary mechanisms and therapeutic opportunities. Front Oncol 9:41.

4. Jackson M, Hassiotou F, Nowak A (2015) Glioblastoma stemlike cells: at the root of tumor recurrence and a therapeutic target. Carcinogenesis 36:177-185.

5. Seymour T, Nowak A, Kakulas F (2015) Targeting aggressive cancer stem cells in glioblastoma. Front Oncol 5:159.

6. Safa AR, Saadatzadeh MR, Cohen-Gadol AA, Pollok KE, Bijangi-Vishehsaraei K (2015) Glioblastoma stem cells (GSCs) epigenetic plasticity and interconversion between differentiated non-GSCs and GSCs. Genes Dis 2:152-163.

7. Cai X, Sughrue ME (2017) Glioblastoma: new therapeutic strategies to address cellular and genomic complexity. Oncotarget 9:9540-9554.

8. Suico MA, Shuto T, Kai H (2017) Roles and regulations of the ETS transcription factor ELF4/MEF. J Mol Cell Biol 9:168177.

9. Sashida G, Liu Y, Elf S, Miyata Y, Ohyashiki K, Izumi M, Menendez S, Nimer SD (2009) ELF4/MEF activates MDM2 expression and blocks oncogene-induced p16 activation to promote transformation. Mol Cell Biol 29:3687-3699.

10. Bazzoli E, Pulvirenti T, Oberstadt MC, Perna F, Wee B, Schultz N, Huse JT, Fomchenko EI, Voza F, Tabar V, Brennan CW, DeAngelis LM, Nimer SD, Holland EC, Squatrito M (2012) MEF promotes stemness in the pathogenesis of gliomas. Cell Stem Cell 11:836-844.

11. Ransohoff JD, Wei Y, Khavari PA (2018) The functions and unique features of long intergenic non-coding RNA. Nat Rev Mol Cell Biol 19:143-157.

12. Shen Y, Liu S, Fan J, Jin Y, Tian B, Zheng X, Fu H (2017) Nuclear retention of the lncRNA SNHG1 by doxorubicin attenuates hnRNPC-p53 protein interactions. EMBO Rep 18:536548.

13. Pruszko M, Milano E, Forcato M, Donzelli S, Ganci F, Di Agostino S, De Panfilis S, Fazi F, Bates DO, Bicciato S, Zylicz M, Zylicz A, Blandino G, Fontemaggi G (2017) The mutant p53-ID4 complex controls VEGFA isoforms by recruiting IncRNA MALAT1.EMBO Rep 18:1331-1351.

14. Vassallo I, Zinn P, Lai M, Rajakannu P, Hamou MF, Hegi ME (2016) WIF1 re-expression in glioblastoma inhibits migration through attenuation of non-canonical WNT signaling by downregulating the IncRNA MALAT1. Oncogene 35:12-21.

15. Kim SS, Harford JB, Moghe M, Rait A, Pirollo KF, Chang EH (2018) Targeted nanocomplex carrying siRNA against MALAT1 sensitizes glioblastoma to temozolomide. Nucleic
Acids Res 46:1424-1440.

16. Gao YL, Zhao ZS, Zhang MY, Han LJ, Dong YJ, Xu B (2017) Long noncoding RNA PVT1 facilitates cervical cancer progression via negative regulating of miR-424. Oncol Res 25:1391-1398.

17. Zhang Y, Yang G, Luo Y (2019) Long non-coding RNA PVT1 promotes glioma cell proliferation and invasion by targeting miR-200a. Exp Ther Med 17:1337-1345.

18. Song T, Yan L, Cai K, Zhao T, Xu M (2018) Downregulation of long noncoding RNA PVT1 attenuates paclitaxel resistance in glioma cells. Cancer Biomark 23:447-453.

19. Chan JJ, Tay Y (2018) Noncoding RNA:RNA regulatory networks in cancer. Int J Mol Sci 19:1310.

20. Shivdasani RA (2006) MicroRNAs: regulators of gene expression and cell differentiation. Blood 108:3646-3653.

21. Han Q, Cheng P, Yang H, Liang H, Lin F (2019) Altered expression of microRNA-365 is related to the occurrence and development of non-small-cell lung cancer by inhibiting TRIM25 expression. J Cell Physiol 234:22321-22330.

22. Huang WC, Jang TH, Tung SL, Yen TC, Chan SH, Wang LH (2019) A novel miR-365-3p/EHF/keratin 16 axis promotes oral squamous cell carcinoma metastasis, cancer stemness and drug resistance via enhancing $\beta 5$-integrin/c-met signaling pathway. J Exp Clin Cancer Res 38:89.

23. Wang Y, Zhang S, Bao H, Mu S, Zhang B, Ma H, Ma S (2018) MicroRNA-365 promotes lung carcinogenesis by downregulating the USP33/SLIT2/ROBO1 signalling pathway. Cancer Cell Int 18:64.

24. Zhu Y, Zhao H, Rao M, Xu S (2017) MicroRNA-365 inhibits proliferation, migration and invasion of glioma by targeting PIK3R3. Oncol Rep 37:2185-2192.

25. Zeng F, Chen H, Zhang Z, Yao T, Wang G, Zeng Q, Duan S, Zhan Y (2018) Regulating glioma stem cells by hypoxia through the Notch 1 and Oct3/4 signaling pathway. Oncol Lett 16:6315-6322.

26. Hambardzumyan D, Bergers G (2015) Glioblastoma: defining tumor niches. Trends Cancer 1:252-265.

27. Miao C, Cao H, Zhang Y, Guo X, Wang Z, Wang J (2018) LncRNA DIGIT accelerates tube formation of vascular endothelial cells by sponging miR-134. Int Heart J 59:1086-1095.

28. Li Z, Liu H, Zhong Q, Wu J, Tang Z (2018) LncRNA UCA1 is necessary for TGF- $\beta$-induced epithelial-mesenchymal transition and stemness via acting as a ceRNA for Slug in glioma cells. FEBS Open Bio 8:1855-1865.

29. Jiang X, Yan Y, Hu M, Chen X, Wang Y, Dai Y, Wu D, Wang Y, Zhuang Z, Xia H (2016) Increased level of H19 long noncoding RNA promotes invasion, angiogenesis, and stemness of 
glioblastoma cells. J Neurosurg 2016:129-136.

30. Mazor G, Levin L, Picard D, Ahmadov U, Carén H, Borkhardt A, Reifenberger G, Leprivier G, Remke M, Rotblat B (2019) The lncRNA TP73-AS1 is linked to aggressiveness in glioblastoma and promotes temozolomide resistance in glioblastoma cancer stem cells. Cell Death Dis 10:246.

31. Xu N, Liu B, Lian C, Doycheva DM, Fu Z, Liu Y, Zhou J, He Z, Yang Z, Huang Q, Zeng H, Guo H (2018) Long noncoding RNA AC003092.1 promotes temozolomide chemosensitivity through miR-195/TFPI-2 signaling modulation in glioblastoma. Cell Death Dis 9:1139.

32. Yang XZ, Cheng TT, He QJ, Lei ZY, Chi J, Tang Z, Liao QX, Zhang H, Zeng LS, Cui SZ (2018) LINC01133 as ceRNA inhibits gastric cancer progression by sponging miR-106a-3p to regulate APC expression and the Wnt/ $\beta$-catenin pathway. Mol Cancer 17:126.

33. Cheng Z, Yu C, Cui S, Wang H, Jin H, Wang C, Li B, Qin M, Yang C, He J, Zuo Q, Wang S, Liu J, Ye W, Lv Y, Zhao F, Yao M, Jiang L, Qin W (2019) circTP63 functions as a ceRNA to promote lung squamous cell carcinoma progression by upregulating FOXM1. Nat Commun 10:3200.

34. Guo Y, Ma D, Jia SF, Liu J, Fan SB, Zhang M, Shi LR, Jiang LL,
Shi JX, Wang HQ, Zheng HY, Li O (2019) [Proliferation of MicroRNA-365 and E74-like factor 4 in cervical cancer cells and its clinical significance]. Zhongguo Yi Xue Ke Xue Yuan Xue Bao 41:220-227.

35. Shang AQ, Wang WW, Yang YB, Gu CZ, Ji P, Chen C, Zeng BJ, Wu JL, Lu WY, Sun ZJ, Li D (2019) Knockdown of long noncoding RNA PVT1 suppresses cell proliferation and invasion of colorectal cancer via upregulation of microRNA-214-3p. Am J Physiol Gastrointest Liver Physiol 317:G222-G232.

36. Chen J, Yu Y, Li H, Hu Q, Chen X, He Y, Xue C, Ren F, Ren Z, Li J, Liu L, Duan Z, Cui G, Sun R (2019) Long non-coding RNA PVT1 promotes tumor progression by regulating the miR143/HK2 axis in gallbladder cancer. Mol Cancer 18:33.

37. Ge Y, Zhou F, Chen H, Cui C, Liu D, Li Q, Yang Z, Wu G, Sun S, Gu J, Wei Y, Jiang J (2010) Sox2 is translationally activated by eukaryotic initiation factor 4E in human glioma-initiating cells. Biochem Biophys Res Commun 397:711-717.

38. Ferletta M, Caglayan D, Mokvist L, Jiang Y, Kastemar M, Uhrbom L, Westermark B (2011) Forced expression of Sox21 inhibits Sox 2 and induces apoptosis in human glioma cells. Int J Cancer 129:45-60. 